\title{
Conflict processing in the anterior cingulate cortex constrains response priming
}

\author{
Bernhard Pastötter *, Simon Hanslmayr, Karl-Heinz T. Bäuml \\ Department of Experimental Dychology, Regensburg University, Unwersitätsstr, 31, 93053 Regensburg, Germany
}

\begin{abstract}
A B S T R A C T
A prominent function of the anterior cingulate cortex (ACC) is to process conflict between competing response options. In this study, we investigated the role of conflict processing in a response-priming task in which manual responses were either validly or invalidly cued. Examining electrophysiological measurements of oscillatory brain activity on the source level, we found response priming to be related to a beta power decrease in the premotor cortex and conflict processing to be linked to a theta power increase in the $A C C$. In particular, correlation of oscillatory brain activities in the ACC and the premotor cortex showed that conflict processing reduces response priming by slowing response time in valid trials and lowering response errors in invalid trials. This relationship emerged on a between subjects level as well as within subjects, on a single trial level. These findings suggest that conlict processing in the ACC constrains the automatic priming process.
\end{abstract}

Motor cortex

Thenta

Beta

Conflict

Response priming

\section{Introduction}

The anterior cingulate cortex (ACC) plays an important role in the adjustment of cognitive control in conflict resolution between relevant and irrelevant information (for a review, see Mansouri et al., 2009). Such cognitive control is needed when goal-irrelevant automatic or prepotent information interferes with goal-relevant information in the selection of the appropriate behavior. For example, the automatic response to extinguish fire is to pour water on the fire source. However, this automatic response is best overridden by throwing table salt, baking soda, or a damp towel on the fire source in the case of an oil fire in the kitchen.

Two current theories of how the ACC processes conflict are the conflict-monitoring theory and the regulatory theory of $A C C$ function. The former theory asserts that the ACC detects and signals conflict to other areas, such as the dorsolateral prefrontal cortex (DLPFC), which then implement cognitive control (Botvinick et al. 2004; Carter and van Veen, 2007). The conflict-monitoring theory assumes a monitoring role for the ACC in a broad range of situations in which conflict might occur from early perceptual to late motor processing. On the other hand, the latter theory asserts that the ACC exerts cognitive control itself and, thus, has a more direct responseregulatory function in resolving conflict (Paus et al., 1998; Posner and Digirolamo, 1998). The regulatory theory is primarily based on evidence that indicates an important role for the ACC in response

\footnotetext{
* Corresponding asthor. Fax: +499419433872.

E-mail address: bentard pastoetieropsychologie.uni regensturg.de (B. Pastöter)
}

selection at the late motor stage. Actually, although the ACC has been shown to signal conflict already from the early perceptual stage of information processing, it is most strongly responsive to conflict at the late motor stage (Milham et al., 2001; van Veen et al., 2001) and post-response conflict due to response errors (Braver et al., 2001; Carter et al., 1998). Consistently, the ACC with its strong connectivity to motor areas has been suggested to be in part a motor structure (Matsumoto et al., 2003; Paus, 2001).

Examining the effects of response conflict and error processing on event-related brain potentials (ERPS), ACC activity has been found to be related to the frontocentral $\mathrm{N} 2$ component and error-related negativity (ERN) (Debener et al., 2005; van Veen and Carter, 2002). In addition, induced frontocentral ACC theta oscillations have been shown to indicate error detection (Luu et al., 2004; Marco-Pallarés et al., 2008; Trujillo and Allen, 2007) and to signal conflict of prepotent responses in the classical Stroop task (Hansimayr et al. 2008) and the Eriksen flanker task (Cavanagh et al., 2009). Moreover, during conflict in prepotent responding, a cross-talk between the ACC and the DIPFC as reflected by long-range theta phase coupling between these brain areas has been found suggesting that when conflict of a prepotent response arises the ACC engages the DLPFC to implement control (Cavanagh et al., 2009; Hanslmayr et al., 2008). In the present response-priming study, we sought to examine the role of theta oscillations in the selection of validly and invalidly primed motor responses.

In response priming, response processing is faster when a target to respond with one hand is shortly preceded by a valid cue that primes the same-hand response and more error-prone when it is preceded by an invalid cue that primes the other-hand response (Sterr and Dean, 2008). Response priming has been studied with neuroimaging 
(Deiber et al, 1996; Lee et al., 1999) and ERPs (Leuthold and Jentzsch, 2002; Mathews et al., 2006). Consistently, lateralized activity in the premotor cortex has been found to be related to response priming. For example, Dehaene et al. (1998) reported that subliminal primes trigger lateralized readiness potentials (LRP) indicating a preparatory activation of the primed response. In addition to response-related evoked activity, there is good evidence that induced beta oscillations in the motor cortex are linked to movement preparation and movement execution (Kaiser et al., 2001; Pfurtscheller and Neuper, 1997). Consistently, in an inhibition-of-return task, beta power in the motor cortex has been found to be responsive both to cue and target presentation (Pastötter et al., 2008). Thereby, induced beta oscillations and evoked readiness potentials are suggested to arise from different neuronal mechanisms providing different information in movement preparation (Shibasaki and Hallett, 2006). In the present study, we sought to examine the role of beta oscillations in response priming.

In the present EEG experiment, a localization-discrimination task was used in which, half-and-half, manual responses were primed by cues or not. Cuing was exogenous (peripheral cues) and uninformative (50\% cue validity). Conflict arose when an actual response (e.g., a left-hand response) had been preceded by an invalid cue (e.g., a cue that primed a right-hand response). We examined induced brain oscillations on the source level and hypothesized that beta power in the premotor cortex is linked to response priming and theta power in the ACC is related to conflict processing. To examine whether conflict processing in the present task affects the priming process, we analyzed the relation of theta activity in the ACC and beta activity in motor sources both between and within subjects.

\section{Materials and methods}

\section{Participants}

Twenty-four right-handed volunteers ( 15 females and 9 males) participated in the study. All participants reported normal or corrected-to-normal vision. Mean age was 23.1 years with a range of 20 to 29 years $(S D=2.0)$. No participant reported any history of neurological disease. All participants gave their written informed consent and received credit points or monetary reward for participation.

\section{Materials and experimental design}

Participants viewed a computer monitor from a distance of $150 \mathrm{~cm}$ and were instructed to maintain fixation on a centrally located gray fixation cross throughout the whole experiment and to not move the eyes. The screen background was black and displayed at all times two gray square outline boxes centered above and below fixation.

After an intertrial interval of variable duration (1950 to $2050 \mathrm{~ms}$ ), 80 of 160 trials began with a $100-\mathrm{ms}$ onset of a cue. The cue consisted of a brightening of one of the two squares and was equally likely to occur at either location. After a variable delay of 50 to $150 \mathrm{~ms}$, the target was presented for $1000 \mathrm{~ms}$. The total cuetarget onset asynchrony thus ranged between 150 and $250 \mathrm{~ms}$. Note that with a cue-target onset asynchrony longer than about $300 \mathrm{~ms}$, the beneficial effect of response priming may be overshadowed by response inhibition (see Pastötter et al., 2008). Intermixed with the cued trials, 80 uncued trials were run in which the preceding intertrial interval was prolongated, respectively (Fig. 1). The target, a red cross, was presented with equal probability within one of the two peripheral boxes with chance coincidence of cue and target locations. Forty trials on which cues and targets were presented at the same location were classified as valid trials, whereas 40 trials on which cues and targets occurred at opposite locations were classified as invalid trials.

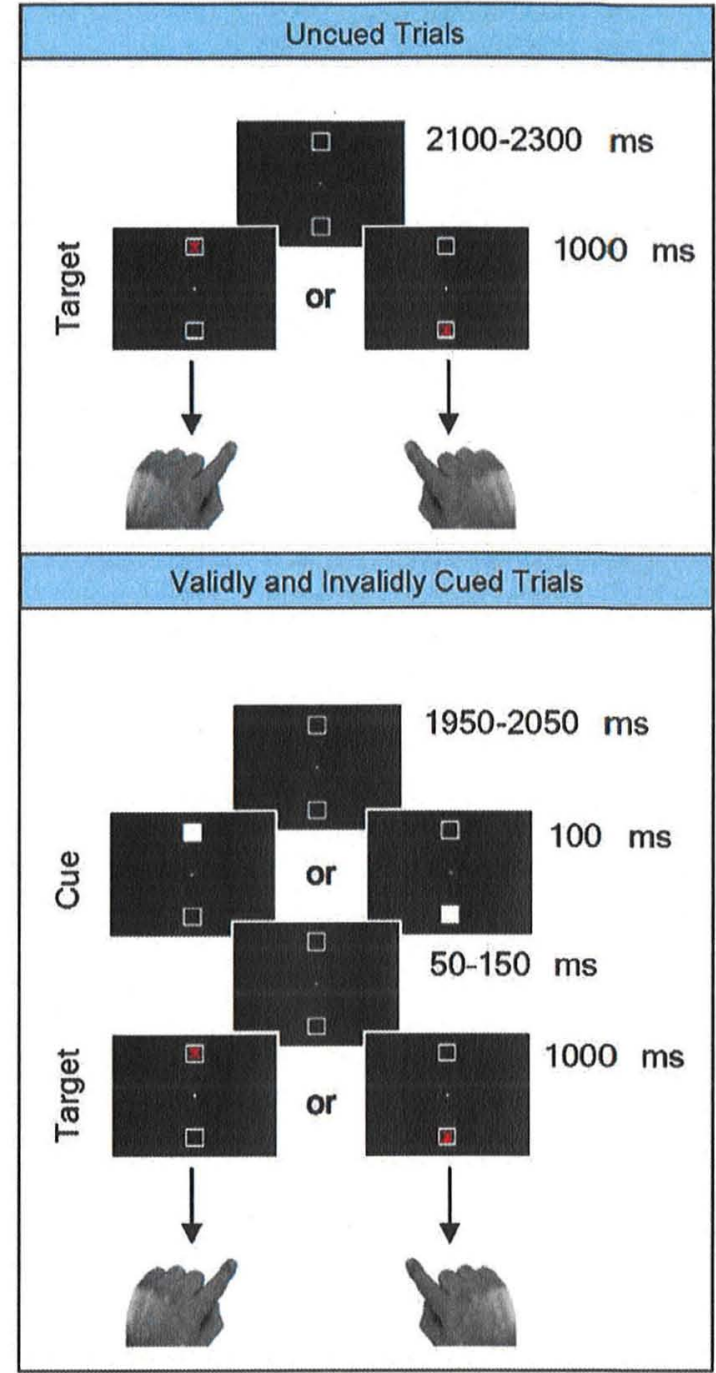

Fig. 1. Participants were instructed to respond to a red cross which was presented in one of the two vertically aligned squares with the index finger of their left hand (targets above fixation) and right hand (targets below fixation) while keeping fixation on the center of the screen. With chance coincidence location, cues (brightening of one of the squares) and targets were presented with equal probability within one of the squares. Both speed and accuracy were stressed in the instruction.

Participants responded based on target's spatial location. Two response keys were marked on the computer keyboard, one on the upper left side and one on the upper right side. Participants were instructed to press the left key with the index finger of their left hand for targets that appeared above fixation and to press the right key with the index finger of their right hand for targets that appeared below fixation but to ignore cue presentation. Both speed and accuracy were stressed in the instruction.

\section{EEG recordings and preprocessing}

EEGs were recorded from $63 \mathrm{Ag} / \mathrm{AgCl}$ scalp electrodes arranged according to the extended 10-10 system and mounted in elastic caps. Vertical and horizontal eye movements were recorded from two additional channels. Electrode $\mathrm{FCz}$ served as common reference. Signals were digitalized with a sampling rate of $500 \mathrm{~Hz}$ and amplified between 3 and $80 \mathrm{~Hz}$ (BrainAmpMR plus, Brain Products). Impedance was kept below $5 \mathrm{k} \Omega$. 
Recordings were re-referenced against average reference and electrooculogram corrected using calibration data to generate individual artifact coefficients (Ille et al., 2002). EEG data were visually inspected for remaining artifacts and segmented into 2000 -ms epochs around target onset. All single trials were time-locked to target onset. Due to the variable cue-target onset asynchrony, cue onset was jittered from -250 to $-150 \mathrm{~ms}$ before target onset. Trials outside the range of 100 to $1000 \mathrm{~ms}$ in reaction time (RT) were excluded from analyses. Mean number of single trials remaining for each participant for analysis was $156.4(\mathrm{SD}=3.8$ ) with a minimum of 146 out of 160 trials.

\section{EEG data analysis}

EEG data were transformed from electrode space to source space (for electrode-space data, see Supplementary material), using the source-analysis module implemented in BESA (Brain Electrical Source Analysis, MEGIS Software v5.1.8). One source was placed in the ACC, one in the left premotor cortex, and one in the right premotor cortex. On the basis of various EEG and brain imaging studies analyzing conflict in information processing (Hanslmayr et al., 2008; Kerns et al., 2004; MacDonald et al., 2000), the ACC source was placed in Brodmann's area 24 (MNI coordinates: $x=0, y=+10, z=+33$ ). Motor sources were placed in Brodmann's area 4 (MNI coordinates: $x=-40 /+40, y=-18, z=+59$ ) which follows localization data of movement-related beta oscillations (Jurkiewicz et al., 2006; Pastötter et al, 2008; Salmelin et al., 1995). In addition, two sources were placed in the left and right DLPFC (Brodmann's area 9; MNI coordinates: $x=$ $-30 /+30, y=+28, z=+56$ ) to analyze phase coupling between the ACC and the prefrontal cortex (see Supplementary material).

Using the short-time Fourier-transformation algorithm implemented in BESA, oscillatory power data were analyzed in a frequency range from 2 to $30 \mathrm{~Hz}$. Time-frequency resolution was set to $50 \mathrm{~ms}$ and $1 \mathrm{~Hz}$ for analysis of lower frequencies in the $\mathrm{ACC}(2$ to $20 \mathrm{~Hz}$ ) and to $25 \mathrm{~ms}$ and $2 \mathrm{~Hz}$ for analysis of higher frequencies in the motor cortex $(10$ to $30 \mathrm{~Hz}$ ). Because BESA decomposes regional source activities into three individual source waveforms corresponding to three equivalent current dipoles oriented radially, vertically, and horizontally, we averaged source activity over dipole orientations. A priori, data were analyzed with and without subtracting the evoked signal from single trials prior to Fourier transformation. As it turned out, the conclusions were the same for the two analysis methods. The present results are based on analyses without subtraction.

To examine differences in event-related power between conditions, we examined stimulus-induced power changes by calculating the percentage of power decrease or power increase in relation to a prestimulus baseline interval (Pfurtscheller and Aranibar, 1977). In our analyses, we focused on the 250 -ms interval following target onset because later effects of response priming and conflict monitoring are presumably overshadowed by movement-execution (as reflected by beta power in the motor cortex) and error-detection activation (as reflected by theta power in the ACC). The prestimulus interval was set from 750 to $500 \mathrm{~ms}$ prior to target onset. This relatively short baseline interval was chosen because reliable beamformer analysis (see below) needs the same duration of target and the baseline interval. A-priori baseline analyses showed that baseline activity in the theta and beta band did not differ between experimental conditions. Finally, to examine whether beta power in the motor cortex was related to theta power in the ACC on a single-trial level, we sorted subjects' single trials based on theta power and split them into a set of high and a set of low theta activity. For each set, we then calculated theta and beta power changes in the underlying sources.

\section{Evaluation of sources}

To evaluate the placing of sources, we used a linearly constrained minimum-variance-vector beamformer implemented in BESA. This method allows to image induced oscillatory activity in a user-defined time-frequency range. In contrast to the source-analysis method, the beamformer method estimates activity in the brain voxel by voxel using the cross-spectral density matrix (Gross et al., 2001). It acts as a spatial filter that estimates the contribution of activity at one point in the brain while minimizing interference from other sources in the brain. Therefore, beamformer analysis shows whether estimated source activity actually arises from a single brain area or may reflect brain activity in distributed brain areas that contribute to estimated source activity. Note that present beamformer analysis accepts the whole volume as solution space including white matter, ventricles, midbrain and deep structures and, therefore, does not allow to draw strong inferences about the exact brain tissue producing an effect in brain activity.

Beamformer analysis was carried out for those frequencies which exhibited significant effects in the analysis on the source level. Percentage of power decrease or power increase was calculated by relating power in the 250 -ms interval following target onset to the baseline interval ranging from 750 to $500 \mathrm{~ms}$ prior to target onset. Plotting of beamformer data was done using self-written Matlab codes (can be requested from the second author) and the Matlab toolbox Fieldtrip (Donders Institute for Brain, Cognition and Behaviour).

\section{Results}

\section{Behavioral results}

\section{Reaction times}

Analyzing mean median RT of participants' responses (Table 1), a two-way analysis of variance (ANOVA) with the within-subject factors CUE (no cue, above, below) and TARGET (above, below) revealed a main effect of $\operatorname{CUE}(F(2,46)=25.6, p<.001)$ and a CUE $\times$ TARGET interaction $(F(2,46)=75.0, p<.001)$, but no main effect of the factor TARGET $(F(1,23)<1)$, the latter showing that right and left hand responses did not differ in RT. Importantly, the interaction arose from faster responses in valid trials than invalid trials $\left(t_{23}=8.7, p<.001\right)$ and uncted trials $\left(t_{23}=9.5, p<.001\right)$. Invalid and uncted trials did not differ in $\mathrm{RT}\left(t_{23}<1\right)$.

\section{Response errors}

Analyzing mean response errors (Table 1), an ANOVA with the factors CUE (no cue, above, below) and TARGET (above, below) revealed marginal main effects of CUE $(F(2,46)=2.9, p=.07)$ and TARGET $(F(1,23)=4.0, p=.06)$, the former due to more errors after cuing than without cuing, the latter due to more response errots with left-hand responses than right-hand responses. Analysis also revealed a CUE $\times$ TARGET interaction $(F(2,46)=13.6, p<.001)$; more errors were committed in invalid trials than in valid trials $\left(t_{23}=4.1, p<.001\right)$ and uncued trials $\left(t_{23}=3.2, p<.01\right)$. Valid and uncued trials did not differ $\left(t_{23}=1.3\right)$. Median-split analysis showed that, compared to low-error subjects, subjects with high error rates in invalid trials were faster in responding to targets in valid trials $\left(t_{22}=2.6, p<.05\right)$.

Table 1

Mean reaction times (RT, standard errors, ms) and localization errors (standard errors, s) as a function of CUE and TAKGET position (relative to fixation).

\begin{tabular}{llrrr}
\hline & & No cue & Cue above & Cue below \\
\hline Target above (left hand response) & RT & $420(7.0)$ & $365(7.7)$ & $437(9.2)$ \\
& Errors & $1.6(0.4)$ & $0.8(0.4)$ & $8.7(2.1)$ \\
Target below (right hand response) & RT & $436(8.3)$ & $429(8.8)$ & $36.4(6.6)$ \\
& Errors & $0.8(0.3)$ & $6.5(1.4)$ & $0.8(0.4)$ \\
\hline
\end{tabular}


Physiological results

Beta activity in the motor cortex

The time course of mean beta activity ( 10 to $28 \mathrm{~Hz}$, see Fig. 2B for significant differences in the time-frequency analysis between cued and uncued trials) in motor sources is plotted in Fig. 2A. The evaluation of source placing by beamformer analysis is shown in Fig. 2C. We found maximum beta activity in the left and right motor cortex, whereas the ACC and the DLPFC did not show specific contribution in beamformer analysis.

Differences in the time course of beta activity between conditions started at target onset with a larger drop in beta power in cued compared to uncued trials. In the $250-\mathrm{ms}$ time interval following target onset, the drop in beta power was largest in valid trials and smallest in uncued trials, with invalid trials in between. Post hoc comparisons showed that differences between conditions were reliable (all $t_{23} s>2.9$, all $p ' s<.01$ ). An ANOVA with the within subjects factors CUING (uncued, valid, invalid) and SOURCE (left, right) revealed a main effect of CUING $(F(2,46)=32.6, p<.001)$, but neither a main effect of SOURCE nor a CUING $\times$ SOURCE interaction $(F \mathrm{~s}<1)$, indicating that the left and right sources were similarly responsive to cuing and validity.

The validity of cues predicted the laterality of beta effects to the targeted movement side. An ANOVA with the within-subjects factors CUING (valid, invalid) and LATERALITY (contralateral, ipsilateral) showed a main effect of CUING $(F(1,23)=8.8, p<.01)$ and a CUING $\times$ LATERALITY interaction $(F(1,23)=4.5, p<.05)$, but no main effect of LATERALITY $(F(1,23)<1)$. The interaction arose from predominant beta ERD contralateral to the targeted movement side in valid trials ( $-15.6 \%$ vs. $-12.2 \%)$, whereas, in invalid trials, beta ERD was more pronounced ipsilateral to the movement side ( $-7.7 \%$ vs. $-10.0 \%)$.

Relating beta activity to behavior, we found a positive correlation of differences in beta power and RT between cued and uncued trials $(r=.58, p<.01)$. Subjects with a larger difference in beta power showed a larger difference in RT. Median-split analysis based on individual differences in beta power between cued and uncued trials revealed that this positive correlation primarily arose from RT differences in the valid cuing condition (Fig. 2D) with faster RTs in the subject group with a strong beta effect compared to the subject group with a weak beta effect $\left(t_{22}=6.0, p<.001\right)$. RTs between groups did not differ in uncued trials $\left(t_{22}<1\right)$ and invalid trials $\left(t_{22}=1.2\right)$. A reliable CUING $\times$ BETA-SPLIT GROUP interaction was found, respectively $(F(2,44)=5.2, p<.01)$.

We also analyzed whether median-split groups differed in the number of response errors. Compared to the subject group with a weak beta effect, the subject group with a strong beta effect committed more errors in invalid trials ( $\tau=.50, p<.01$, nonparametric test). We did not analyze differences in response errors between groups in valid and uncued trials because error rates were too small and invariant in these conditions. Together, these results indicate that response priming with faster RTs in valid trials and more response errors in invalid trials is reflected by beta activity in the motor cortex.

\section{Theta activity in the $A C C$}

The time course of mean theta activity ( 4 to $8 \mathrm{~Hz}$, see Fig. 3B for significant differences in the time-frequency analysis of validly and invalidly cued trials) in the ACC source is plotted in Fig. 3A. The evaluation of source placing by beamformer analysis is shown in Fig. $3 C$. We found maximum theta activity in the ACC, whereas the motor cortex and the DLPFC did not show specific contribution in beamformer analysis.

Differences in the time course of theta activity after valid and invalid cuing started at target onset with a larger increase of theta power in invalidly compared to validly cued trials. In the 250 -ms time interval following target onset, the increase in theta power in the invalid condition was reliably larger than in the valid condition $\left(t_{23}=4.7, p<.001\right)$.

Relating theta activity to behavior, we found a negative correlation of differences in theta power and RT between invalid and valid trials $(r=-.64, p<.001)$. Subjects with a larger difference in theta power showed a smaller difference in RT. Median-split analysis based on individual differences in theta power between invalid and valid trials revealed that this negative correlation primarily arose from RT differences in the valid cuing condition (Fig. 3D) with slower RTs in the subject group with a strong theta effect compared to the subject group with a weak theta effect $\left(t_{22}=2.8, p<.01\right)$. RTs between groups
A

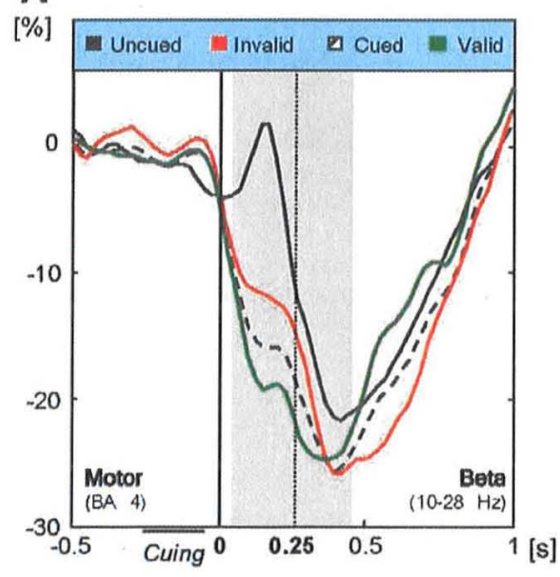

B

$[\mathrm{Hz}]$

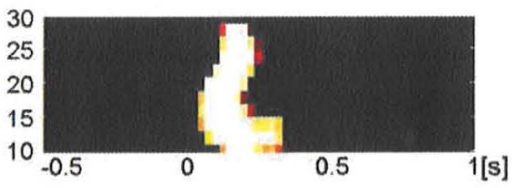

C

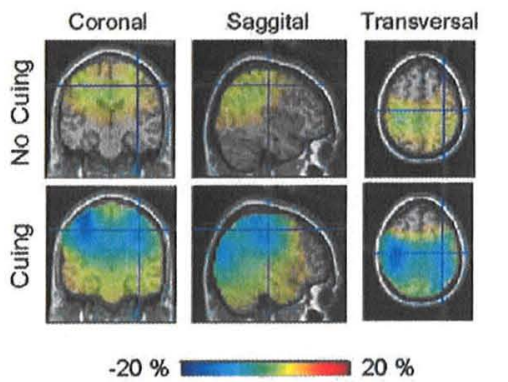

D

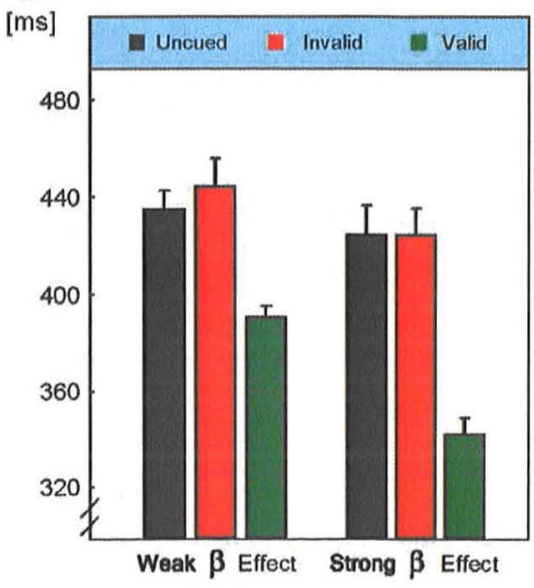

Fig. 2. (A) Time course of beta power ( 10 to $28 \mathrm{~Hz}$ ) in the motor cortex. time-locked to target onset and averaged across hemispheres as the left and right sources were similarly responsive to cuing and validity. The dashed line shows beta power in cued trials (average of valid and invalid trials). The gray bar indicates time windows of significant differences between cued and uncued trials ( $p^{\prime} s<.05$; based on point-by-point Wilcoxon signed-rank tests for 25 -ms time windows). We focused on the 250 -ms interval following target onset because later effects may have been overshadowed by effects of movement execution. (B) Significant differences in beta power as a factor of cuing in the time-frequency plot, timelocked to target onset $(\boldsymbol{p}<.01)$. (C) Beamformer analysis confirmed placing of beta sources in the motor cortex. Beta power decreases (cold color coding) were more pronounced in cued trials than in uncued trials. Differences were restricted to the motor cortex. (D) Behavioral split data based on beta effects as a factor of cuing. In valid trials, subjects with a strong beta effect responded faster than subjects with a weak beta effect $(p<.001)$. 

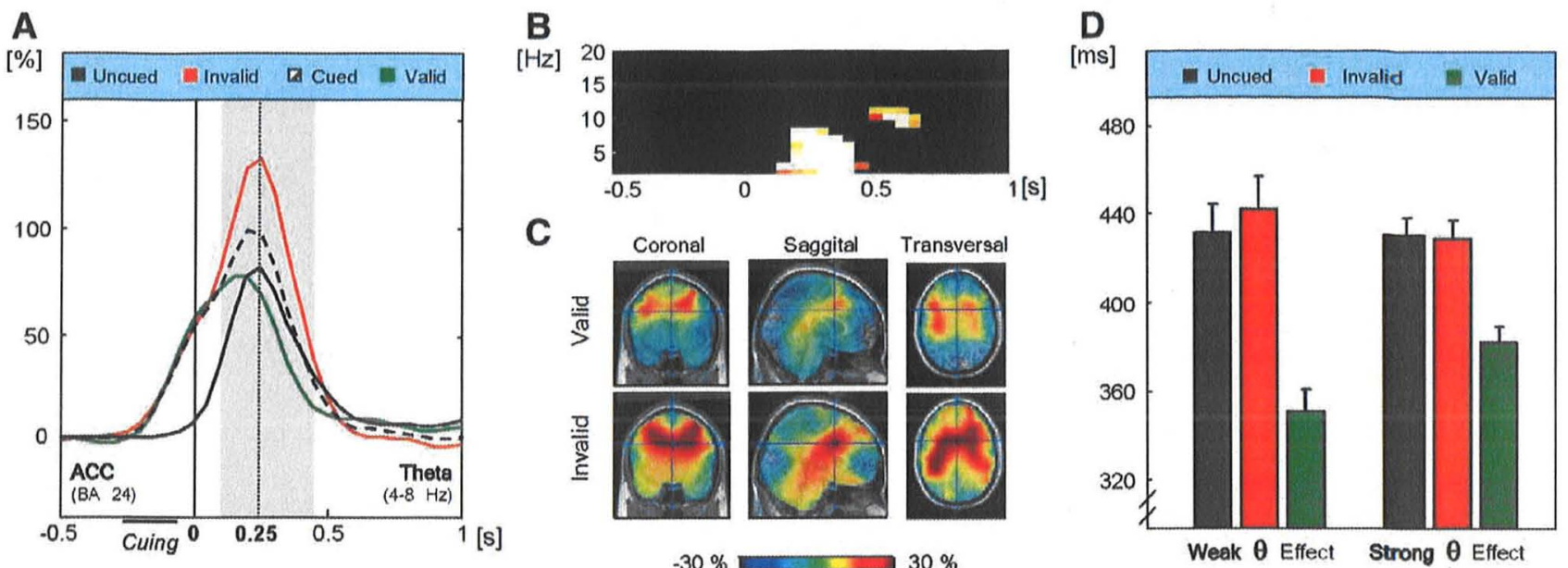

Fig. 3. (A) Time course of theta power ( 4 to $8 \mathrm{~Hz}$ ) in the ACC, time-locked to target onset. The dashed line shows theta power in cued trials (average of valid and invalid trials). The gray bar indicates time windows of significant differences between valid and invalid trials ( $p^{\prime} s<.05$; based on point-by-point Wilcoxon signed-rank tests for 50 -ms time windows). We focused on the 250-ms interval following target onset because later effects may have been overshadowed by effects of error-detection in invalid trials. (B) Significant differences in theta power as a factor of cue validity in the time-frequency plot, time-locked to target onset $(\boldsymbol{p}<.01)$. Significant post-response differences in alpha power ( 8 to $12 \mathrm{~Hz}$ ) were not further analyzed as we focused on brain activity preceding participants' actual responses. (C) Beamformer analysis confirmed placing of the theta source in the ACC. Theta power increases (warm color coding) were more pronounced in invalid trials than in valid trials. Differences were restricted to the ACC. (D) Behavioral split data based on theta effects as a factor of cue validity. In valid trials, subjects with a strong theta effect responded more slowly than subjects with a weak theta effect $(p<.01)$.

did not differ in uncued trials and invalid trials $\left(t_{22}\right.$ 's $\left.<1\right)$. We found a CUING $\times$ THETA-SPLIT GROUP interaction, respectively $(F(2,44)=$ $6.5, p<.01)$.

We also analyzed whether median-split groups differed in the number of response errors. Compared to the subject group with a weak theta effect, the subject group with a strong theta effect committed less errors in invalid trials $(T=-.50, p<.01$, nonparametric test). Again, we did not analyze differences in response errors in valid and uncued trials. Together, these results indicate that theta activity in the ACC is negatively related to response priming by slowing response time in valid trials and lowering response errors in invalid trials.

\section{Relation between ACC and motor activity}

Our last step was to correlate theta power in the ACC with beta power in the motor cortex separately for conditions both across and within subjects. Across subjects, we found a positive correlation between theta and beta power both after valid cuing $(r=.69, p<.001)$ and invalid cuing $(r=.63, p<.01)$. Thus, a large increase of theta power in the ACC was accompanied by a small drop of beta power in the motor cortex (Fig. 4A). In contrast, without cuing, no such relation was observed $(r=.10, p=.66)$. Crucially, the nonfinding of a correlation in uncued trial was not due to a restricted range of variability in this condition as a comparisons of variance in theta and beta power between uncued and valid trials revealed no significant differences $\left(t_{22}\right.$ 's $\left.<1.3\right)$. On the other hand, in invalid trials we found the range both of theta and beta power to be significantly larger than in valid and uncued trials (all $t_{22}$ 's $>2.2$, all $p$ 's $<.05$ ).

Within subjects, median-split analysis based on single-trial thet power in the ACC also revealed a positive relation to beta power in the motor cortex, both in valid and invalid trials (Fig. 4B). In single trials, a larger increase of theta power in the ACC was related to a smaller drop of beta power in the motor cortex in the same trial. In addition, a median single-trial analysis of RT as a factor of VALIDITY (valid trial, invalid trial) and single-trial THETA POWER in the ACC (large, small) revealed a main effect of VALIDITY $(F(1,23)=81.0, p<.001)$ and a main effect of THETA POWER $(F(1,23)=6.0, p<.05)$ but no interaction between factors $(F(1,23)<1)$. In fact, RT was faster in small-theta-power trials in the ACC (and small-beta-power trials in the motor cortex) than in large-theta-power trials in the ACC (and large-beta-power trials in the motor cortex). This relationship was found for valid and invalid trials ( $361 \mathrm{~ms} v$ s. $372 \mathrm{~ms}$, and $424 \mathrm{~ms}$ vs. $433 \mathrm{~ms}$, respectively). Taken together, theta power in the ACC predicted beta power in the motor cortex and RT. This relationship was evident across subjects and within subjects on a single trial level, suggesting that ACC activity is directly linked to motor processing in response priming.

\section{Discussion}

The goal of the present study was to examine the role of conflict processing in response priming with electrophysiological measurements of oscillatory brain activity. Consistent with prior behavioral work, we found response processing to be faster after valid cuing and more error-prone after invalid cuing. In line with prior physiological work, response processing was related to oscillatory beta activity in the motor cortex and conflict processing was linked to theta activity in the ACC. Going beyond the prior work, the present results show that conflict processing in the ACC constrains response priming in the motor cortex by slowing response processing in valid trials and lowering response errors in invalid trials.

Consistent with prior imaging and ERP studies, the analysis of brain oscillations showed that response priming is related to lateralized activity in the motor cortex. In particular, the present results demonstrate that response priming is reflected by a decrease of beta power. This finding is well in line with the idea that beta power decrease in the motor cortex is rather related to movement preparation than to movement execution. Corresponding evidence comes from studies in which a beta decrease has been observed in motor imagery, no-go tasks, and delayed responding (Kaiser et al., 2001; Leocani et al., 2001; Pfurtscheller and Neuper, 1997). Together with our previous study in which we related response inhibition to an increase of beta power (Pastötter et al., 2008), the present results indicate that response priming and response inhibition are related to beta power in the opposite direction. Accordingly, beta power changes in motor cortex seem to reflect the decrease (like in response priming) or increase (like in response inhibition) of the distance from baseline activity to motor response threshold. 
A

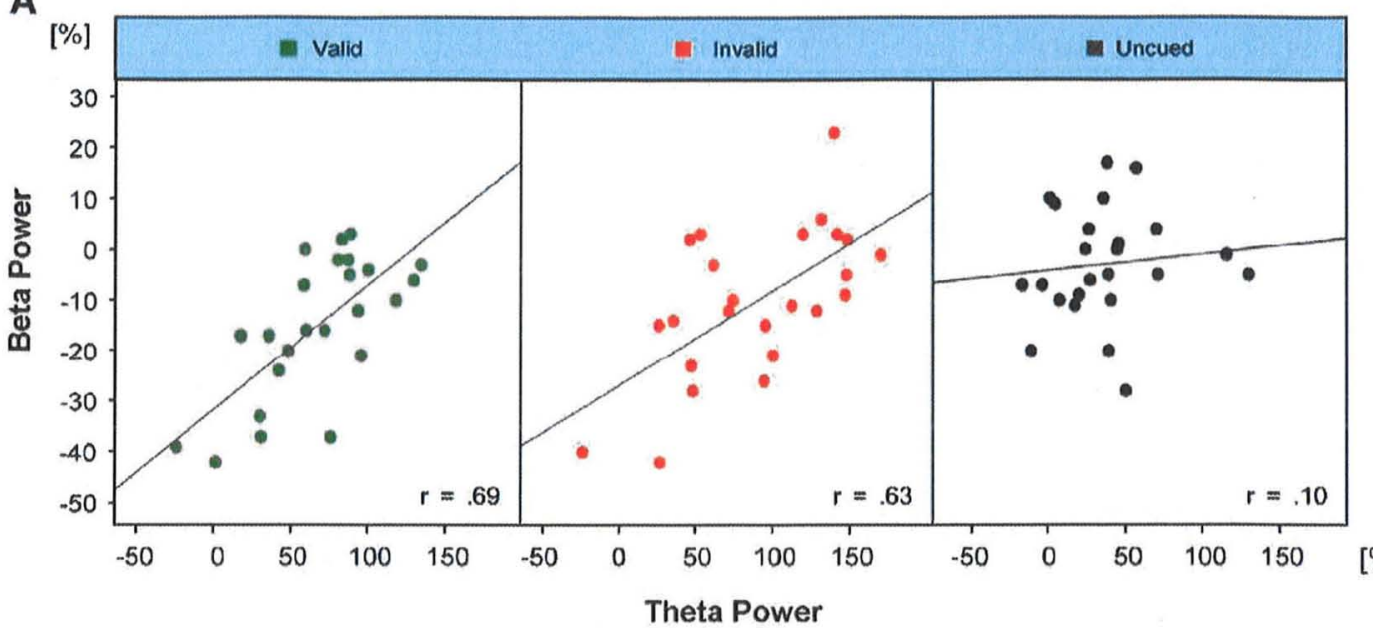

B
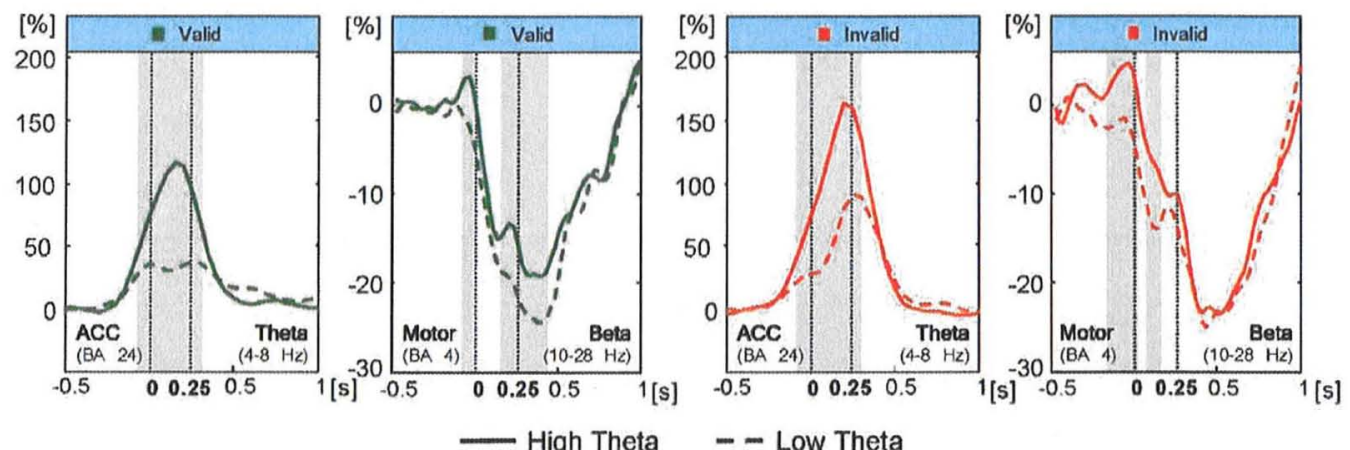

High Theta

- - Low Theta

Fig. 4. (A) Between-subjects correlational analysis. In valid and invalid trials, theta power in the ACC and beta power in the motor cortex were positively related ( $\boldsymbol{p}$ 's $<.01$ ). No such relation was found without cuing. (B) Within-subjects correlational analysis. Median-split analysis based on single trial theta power in the ACC revealed significant differences in beta power in the motor cortex both in valid and invalid trials. Gray bars indicate time windows of significant differences ( $p$ 's $<.05$; based on point-by-point Wilcoxon signed-rank tests for 50-ms time windows in the theta analyses and 25 -ms time windows in the beta analyses).

Conflict processing in the $\mathrm{ACC}$ has been suggested to be beneficial for the processing of less potent responses in various paradigms, including the Stroop task (Kerns et al., 2004; MacDonald et al., 2000), the Eriksen flanker task (Botvinick et al., 1999), and the Simon task (Peterson et al., 2002). In the present response-priming task, we found that processing of (potential) conflict in the ACC affects response behavior. Conflict processing in the ACC as reflected by theta activity slowed the processing of validly cued responses and reduced response errors of invalidly cued responses. ${ }^{1}$ Correlational analyses showed that theta power in the ACC predicted beta power in the motor cortex in cued but not in uncued trials. This relationship was found for both invalid and valid trials suggesting that, just like conflict arises in invalid trials, potential conflict processing arises in valid trials. In fact, potential conflict seems reasonable because cuing is nonpredictive and, in contrast to paradigms in prepotent responding, precedes target presentation. In addition to between-subjects analyses, we found that single-trial theta activity in the ACC was positively related to singletrial beta activity in the motor cortex and predicted RT. Thus, in contrast to prepotent responding, in which executive control has been

\footnotetext{
${ }^{1}$ Arguably, compared to responding without cuing, we did not observe an increase in RT in invalid trials. This nonfinding is most likely due to the positive effect of moto preparation in cued trials masking the detrimental RT effect of invalid cuing. On the other hand, not finding a beneficial effect on accuracy after valid cuing apparently is due to a floor effect in response errors in uncued trials. In future experiments, by including an additional neutral cuing condition in which both responses are cued and by increasing error probability, these effects should show up.
}

suggested to be exerted on the next trials following conflict via the DLPFC (Botvinick et al., 2004; Carter and van Veen, 2007), in the present response-priming task, the $\mathrm{ACC}$ seems to regulate conflict itself within the same trial (Paus et al., 1998; Posner and DiGirolamo, 1998). Accordingly, ACC processing may increase the motor response threshold leading to slower responses in valid trials and less response errors in invalid trials.

In prepotent responding, the $\mathrm{ACC}$ is meant to engage the DLPFC to implement top-down control in the service of goal-directed processing of less potent responses (Carter and van Veen, 2007; Miller, 2000). Thereby, conflict processing in the ACC and strategic control in the DLPFC has been shown to be dissociable (Bunge et al., 2002; MacDonald et al., 2000). In the present task, we did not find the DLPFC to be responsive to signaling of conflict in the ACC. Theta phase coupling between the ACC and the prefrontal cortex, which has been observed in cognitive control of prepotent responses (Cavanagh et al., 2009; Hanslmayr et al., 2008), was not affected by cue validity in the present response-priming task suggesting that subjects did not adapt task engagement but retained their dominant strategy throughout the session. On the other hand, we found that cuing per se led to an increase of theta phase coupling between the ACC and the right DLFPC with cue onset (see Supplementary material). This cue-induced increase of theta phase coupling may refer to a cross-talk between executive control structures about the dominant strategy on how cue information is processed (e.g., stressing speed or accuracy). If true, covariance of brain activities may be linked to between-subjects differences in the speed-accuracy tradeoff (Bogacz et al., 2010). 
However, with the present data, this possibility cannot be tested. Future work examining the possible role of task engagement by stressing either speed or accuracy is eligible.

In sum, the present findings indicate that conflict processing in response priming reduces both the positive speed effect of valid cuing and the detrimental accuracy effect of invalid cuing. As reflected by theta and beta brain oscillations in the ACC and the motor cortex. increased conflict processing is related to reduced response priming with slower RTs in valid trials and less errors in invalid trials. Together, the present results suggest that cognitive control can constrain the automatic priming process.

\section{Acknowledgments}

The authors thank two anonymous reviewers for their helpful suggestions on a previous version of the manuscript.

\section{Appendix A. Supplementary data}

Supplementary data associated with this article can be found, in the online version, at doi: $10.1016 /$ j.neuroimage 2010.01 .095

\section{References}

Bogacz, R., Wagenmakers, E.J. Forstmann, B.U. Nieuwenhuis, S., 2010. The neural basis of the speed-accuracy tradeoff. Trends Neurosci, 33, 20-16.

Botvinick, MM., Nystrom, L.E. Fissell, K., Carter, C.S., Cohen, J.D. 1999. Conflict monitoring versus selection-for-action in anterior cingulate correx. Nature 402 $179-181$.

Botvinick, MM., Cohem, J.D., Carter, C.5., 2004. Confict monitoring and anterior cingulate cortex: an update. Trends cogn Sci. 8. 539-546.

Braver, T.S., Barch, D.M. Gray, J.R., Molfese, D.I, Snyder. A. 2001. Anterior cingulate cortex and response confict: effects of frequency, inhibition and errors. Cereb. Cortex 11, 825-836

Bunge, S.A., Hazeltine, E., Scanlon, M.D. Rosen, A.C., Gabrieli, J.D.E, 2002. Dissociable contributions of prefrontal and parietal cortices to response selection. Neurolmage $17,1562 \cdots 1571$

Carter, C.S., van Veen, V. 2007. Anterior cingulate cortex and confict detection: an update of theory and data. Cogn. Affect. Behav. Neurosci, 7, 367-379.

Carter, C. S., Braver. T.S., Barch, D.,., Bowinick, MM., Noll, D., Cohen, J.D., 1998. Anterior cingulate cortex, error detection, and the online monitoring of perfomance science $280,747-749$.

Cavanagh, J.F, Cohen, M,X, Allen, J., 2009. Prelude to and resolution of an error: EEC phase synchrony reveals cognitive control dynamics during action monitoring 1. Neurosci. 29, $98-105$

Debener, S., Ulisperger, M., Siegel, M. Fiehler, K., von Cramon, D.Y., Engel, A.K., 2005 Trial-by-trial cospling of concurrent electroencephalogram and functiona magnetic resonance imaging identifes the dynamics of performance monitoring J. Neurosci. $25,11730-11737$.

Dehaene, S., Naccache, L., Le ClecH, G. Koechlin, E, Mueller, M., Dehaene-Lambertz, G. van de Moortele, P.F., Le Bihan, D., 1998. Imaging unconscious semantic priming Nature $395,597-600$.

Deiber, MP., Ibañez V., Sadato, N., Hallett. M., 1996. Cerebral structures participating in motor preparation in humans: a positron emission tomography study J. Neurophysiol. $75,233-247$

Gross, J., Kujala, J, Kamalainen, M., Timmermann, L., Schnitaler, A., 5almelin, R., $200 \mathrm{I}$ Dynamic inaging of coherent sources: Studying neural interactions in the human brain. Proc. Natl. Acad. Sci. U. S. A. 98, 694-699.

Hanstmayr, S., Pastötter, B. Bäuml, K.-H., Gruber, S., Wimber, M., Klimesch, W., 2008 The electrophysiological dynamics of interference during the Stronp task J. Cogn Neturosci. $20,215-225$

Ille, N., Berg. P., Scherg, M., 2002. Artifact correction of the ongoing EEG using spatia filters based on artifact and brain signal ropographies. J. Clin. Neurophysiol. 19 $113-124$
Jurkiewicz, M.T. Gatz, W.C., Bostan, A.C. Cheyne, D. 2006. Post-movement beta re bound is generated in motor cortex: evidence from neuromagnetic recordings. Neurolmage $32,1281-1289$

Kaiser, J, Birbaumer, N., Lutzenberger, W., 2001. Event-related beta desynchronization indicates timing of response selection in a delayed-response paradigm in humans. Neurosci. Lett. $312,149-152$.

Kerns, J.G., Cohen, J.D., MacDonald, A.W., Cho, R.Y., Stenger, V.A., Carter, CS., 2004 Anterior cingulate conflict monitoring and adjustments in control. Science 303. $1023-1026$.

lee, K.M., Chang, K.H., Roh, J.K., 1999. Subregions within the supplementary motor area activated at different stages of movement preparation and execution. Neurolmage $9,117-123$.

Leocani, L., Toro, C, Zhuang, P., Gerloff, C., Hallet,, M., 2001. Event-related desychronization in reaction time paradigms: a comparison with event-related potentials and corticospinal excitability. Clin. Neurophysiol. 122, 923-930.

Leuthold, $H$., Jentzsch, 1., 2002. Distinguishing neural sources of movement preparation and execution. An electrophysiological analysis. Biol. Psychol. 60,179-198.

Lui, P.. Tucker, D.M. Makeig, S., 2004. Frontal midline theta and the error related negatwiy: Neurophysiological mechanisms of action regulation. Clin. Neurophysiol $115,182,-1835$

MacDonald, AW., Cohen, J.D., Stenger, V.A., Carter, CS, 2000. Dissociating the role of the dorsolateral prefrontal and anterior cingulate cortex in cognitive control Science 288, 1835-1838.

Mansouri, F.A., Tanaka, K., Buckley, M.!., 2009. Conflict-induced behavioural adjust ment: a clue to the executive functions of the prefrontal cortex. Nat. Rev., Neurosi $10,141-152$.

Marco-Pallarés, J., Camara, E., Münte, T.F., Rodriguez-Fornells, A., 2008. Neural mechanisms underiying adaptive actions after slips. I. Cogn. Neirosci. 20.1595-1610.

Mathews, S., Dean, P.J.A. Sterr, A, 2006. EEG dipole analysis of motor-priming foreperiod activity reveals separate sources for motor and spatial attention components. Clin. Neurophysiol. 117, 2675-2683

Matsumoto, K.. Suzuki. W. Tanaka, K., 2003. Neuronal correlates of goal-based noto selection in the prefrontal cortex. Science 301, 229-232

Mitham, M.P., Banich, M.T., Webb, A. Barad, V., Cohen, N.., Wszalek, T., Kramer, A.F 2001. The relative involvement of anterior cingulate and prefrontal cortex in attentional control depends on nature of conflict. Brain Res. Cogn. Brain Res. 12 467-473.

Miller, E.K., 2000. The prefrontal cortex and cognitive control. Nat Rev., Neurosci. 1 $59-65$

Pastötter, B., Hanslmayl. S., Bäuml, K.H., 2008. Inhibition of return arises from inhibition of response processes: an analysis of oscillaton beta activity. J. Cogn. Netrosci. 20 . $65-75$

Paus, T. 2001. Primate anterior cingulate cortex: where motor control, drive and cognition interface. Nat. Rev. Neurosci. 2. 417-424.

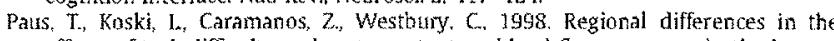
effects of task diffculy and motor output on blood fow response in the human anterior cingulate cortex: a review of 107 PET activation studies. Neurokeport 9 , R37-R47.

Peterson, B.S Kane, M.J. Alexander, G.M., Lacadie, C. Skudlarski, P., Leung, H.C. May, J. Gore, I.C., 2002. An event-related functional MRI study comparing interference effects in the Simon and Stroop tasks. Brain Res. Cogn. Brain Res. 13. $427-440$.

Pfurtscheller, G., Aranitar, A., 1977. Event-related cortical desynchronization detected by power measurements of scalp EEG. Electroencephalogr. Clin. Neurophysiol. 42, $817-826$

Pfurtscheller, $G$, Neuper, $C$., 1997. Motor imagery activates primary sensorimotor area in humans. Neirosci. Leti. 235, $65-68$.

Posner, M.I., DiGirolamo, G., 1998. Executive attention: conflict, target decection and cognitive control. In: Parasuraman, $R$. (Ed.), The Attentive Brain. MiT Press. Cambridge, pp. 401-423.

Salmelin, R. Hamalainen, M. Kajola, M., Hari, R, 1995. Functional segregation of movement-related rhythmic activity in the human brain. Neuromage 2 , $237-243$.

Shibasaki, H. Hallett, M., 2006. What is the Bereitschafts potential? Cin. Neurophysiol $117,2341-2356$

Sterr. A., Dean, P.I.A. 2008. Neural correlates of movement preparation in healthy ageing. Eur. J. Neurosci. 27, 254-260.

Trujillo. LT. Allen, JJ.B., 2007. Theta EEG dynamics of the enror-related negativity. Clin Neurophysiol. 118, 645-668

van Veen, V. Carter, CS, 2002. The timing of action-monitoring processes th the anterior cingulate cortex. J. Cogn. Neurosci. $14,593-602$

yan Veen, V., Cohen, J.D., Botvinick, M.M., Stenger, V.A., Carter, C.S., 200\%. Anterior cingulate cortex, conflict monitoring, and levels of processing. Netromage 14 $1302-1308$. 